\title{
A Note on Lax Pairs of the Sawada-Kotera Equation
}

\author{
Sergei Sakovich \\ Institute of Physics, National Academy of Sciences of Belarus, 220072 Minsk, Belarus \\ Correspondence should be addressed to Sergei Sakovich; saks@tut.by
}

Received 30 May 2014; Accepted 19 August 2014; Published 24 August 2014

Academic Editor: Abdul Hamid Kara

Copyright (C) 2014 Sergei Sakovich. This is an open access article distributed under the Creative Commons Attribution License, which permits unrestricted use, distribution, and reproduction in any medium, provided the original work is properly cited.

We prove that the new Lax pair of the Sawada-Kotera equation, discovered recently by Hickman, Hereman, Larue, and Göktaş, and the well-known old Lax pair of this equation, considered in the form of zero-curvature representations, are gauge equivalent to each other if and only if the spectral parameter is nonzero, while for zero spectral parameter a nongauge transformation is required.

\section{Introduction}

Recently, the following interesting result was obtained by Hickman et al. [1]. It turned out that the Sawada-Kotera equation $[2,3]$

$$
u_{t}+5 u^{2} u_{x}+5 u_{x} u_{x x}+5 u u_{x x x}+u_{x x x x x}=0
$$

possesses two different Lax representations in the operator form

$$
L \psi=\lambda \psi, \quad \psi_{t}=M \psi,
$$

where subscripts of the scalar functions $u$ and $\psi$ denote respective derivatives, $L$ and $M$ are linear differential operators expressed in powers of the derivative operator $D_{x}$, and $\lambda$ is the spectral parameter. The first Lax pair, given by the operators

$$
\begin{gathered}
L_{1}=D_{x}^{3}+u D_{x} \\
M_{1}=9 D_{x}^{5}+15 u D_{x}^{3}+15 u_{x} D_{x}^{2}+\left(5 u^{2}+10 u_{x x}\right) D_{x}
\end{gathered}
$$

is well known $[4,5]$. The second Lax pair, given by the operators

$$
\begin{gathered}
L_{2}=D_{x}^{3}+u D_{x}+u_{x}, \\
M_{2}=9 D_{x}^{5}+15 u D_{x}^{3}+30 u_{x} D_{x}^{2} \\
+\left(5 u^{2}+25 u_{x x}\right) D_{x}+\left(10 u u_{x}+10 u_{x x x}\right),
\end{gathered}
$$

is new, in the sense that it appeared in [1] for the first time in the literature.

Many experts, according to their private communications, noticed that the second Lax pair (4) is related to the first Lax pair (3) by the transformation

$$
L_{2}=-L_{1}^{\dagger}, \quad M_{2}=-M_{1}^{\dagger},
$$

where the dagger denotes the Hermitian conjugate. This transformation (5) always turns a Lax pair of an integrable equation into a Lax pair of the same equation, but usually the resulting Lax pair has essentially the same form as the original one (we believe that for this reason no second Lax pair was discovered in [1] for the Kaup-Kupershmidt equation, in particular). Let us note, however, that the Lax pairs (3) and (4) are different in form. Some other experts, also according to their private communications, noticed that the old Lax pair (3) and the new one (4) are related to each other by the transformation

$$
L_{2}=D_{x} L_{1} D_{x}^{-1}, \quad M_{2}=D_{x} M_{1} D_{x}^{-1},
$$

which corresponds to the transformation $\psi \mapsto \psi_{x}$ made in (2). Thus, there exist (at least) two different ways to relate the Lax pairs (3) and (4) to each other, and we believe that this point deserves further investigation using more general description of Lax pairs than their operator form.

In the present paper, we study these two Lax pairs of the Sawada-Kotera equation (1) - the old one, (2) with (3), and the new one, (2) with (4)-in the matrix form

$$
\Phi_{x}=X \Phi, \quad \Phi_{t}=T \Phi,
$$


or, what is the same, in the form of zero-curvature representations (ZCRs)

$$
D_{t} X-D_{x} T+[X, T]=0
$$

where $\Phi(x, t)$ is a three-component column vector, $X$ and $T$ are $3 \times 3$ matrices, and the square brackets denote the matrix commutator. In Section 2, we show that, for any nonzero value of the spectral parameter, the new Lax pair of the Sawada-Kotera equation and the old one are related to each other by a gauge transformation of ZCRs

$$
\begin{gathered}
\Phi \longmapsto G \Phi, \quad \operatorname{det} G \neq 0, \\
X \longmapsto G X G^{-1}+\left(D_{x} G\right) G^{-1}, \\
T \longmapsto G T G^{-1}+\left(D_{t} G\right) G^{-1},
\end{gathered}
$$

where $G$ is a $3 \times 3$ matrix. In Section 3, we show that, for any value of the spectral parameter including zero, the new Lax pair and the old one are related to each other by a gauge transformation (9) combined with a different type of equivalence transformations of ZCRs (8); namely,

$$
X \longmapsto-\widetilde{X}, \quad T \longmapsto-\widetilde{T},
$$

where the tilde denotes the matrix transpose. Section 4 contains concluding remarks.

We use computationally effective techniques, such as the method of gauge-invariant description of ZCRs, developed in $[6,7]$ independently, and the method of cyclic bases of ZCRs $[7,8]$, and follow the terminology and notations adopted in [8].

\section{Nonzero Spectral Parameter}

Introducing the three-component column vector

$$
\Phi=\left(\begin{array}{c}
\psi \\
\psi_{x} \\
\psi_{x x}
\end{array}\right)
$$

we can rewrite the Lax pairs (2) with the operators (3) and (4) in their matrix form (7). The old Lax pair of the Sawada-Kotera equation, determined by the operators (3), corresponds to the ZCR (8) with the matrices

$$
\begin{gathered}
X_{1}=\left(\begin{array}{ccc}
0 & 1 & 0 \\
0 & 0 & 1 \\
\lambda & -u & 0
\end{array}\right), \\
T_{1}=\left(\begin{array}{ccc}
6 \lambda u & -u^{2}+u_{x x} & 9 \lambda-3 u_{x} \\
9 \lambda^{2}+3 \lambda u_{x} & -3 \lambda u+v & -u^{2}-2 u_{x x} \\
-\lambda u^{2}+\lambda u_{x x} & 9 \lambda^{2}+u^{3}+2 u u_{x x}+v_{x} & -3 \lambda u-v
\end{array}\right),
\end{gathered}
$$

where $\lambda$ denotes the spectral parameter, and

$$
v=u_{x x x}+u u_{x}
$$

The new Lax pair of the Sawada-Kotera equation, (2) with (4), corresponds to the ZCR (8) with the matrices

$$
\begin{gathered}
X_{2}=\left(\begin{array}{ccc}
0 & 1 & 0 \\
0 & 0 & 1 \\
\mu-u_{x} & -u & 0
\end{array}\right), \\
T_{2}=\left(\begin{array}{ccc}
6 \mu u+3 u u_{x}+v & -u^{2}-2 u_{x x} & 9 \mu+3 u_{x} \\
9 \mu^{2}+3 u u_{x x}+v_{x} & -3 \mu u-v & -u^{2}+u_{x x} \\
p & q & -3 \mu u-3 u u_{x}
\end{array}\right),
\end{gathered}
$$

where $\mu$ stands for the spectral parameter, $v$ is given by (13), and

$$
\begin{gathered}
p=-\mu u^{2}-2 u^{2} u_{x}+\mu u_{x x}+2 u_{x} u_{x x}+3 u v+v_{x x} \\
q=9 \mu^{2}+u^{3}-3 \mu u_{x}+2 u u_{x x} .
\end{gathered}
$$

We have changed the notation for the spectral parameter in (14) because no relation between the parameters of (12) and (14) is assumed initially.

Let us compute the cyclic bases $[7,8]$ of the ZCRs (8) with the matrices (12) and (14), in order to see if there are any obstacles to relate these two ZCRs by a gauge transformation

$$
\begin{gathered}
X_{2}=G X_{1} G^{-1}+\left(D_{x} G\right) G^{-1}, \\
T_{2}=G T_{1} G^{-1}+\left(D_{t} G\right) G^{-1} .
\end{gathered}
$$

For the matrix $X_{1}$ given by (12) with a nonzero spectral parameter $\lambda \neq 0$, we find that the cyclic basis is eight-dimensional, consisting of the matrices $C_{1}, \nabla_{1} C_{1}, \nabla_{1}^{2} C_{1}, \ldots, \nabla_{1}^{7} C_{1}$, where $C_{1}$ is the characteristic matrix,

$$
C_{1}=\frac{\partial X_{1}}{\partial u}=\left(\begin{array}{ccc}
0 & 0 & 0 \\
0 & 0 & 0 \\
0 & -1 & 0
\end{array}\right)
$$

and the covariant derivative $\nabla_{1}$ is defined by the relation $\nabla_{1} A=D_{x} A-\left[X_{1}, A\right]$ with any $3 \times 3$ matrix $A$. The closure equation of the cyclic basis,

$$
\nabla_{1}^{8} C_{1}=a_{0} C_{1}+a_{1} \nabla_{1} C_{1}+a_{2} \nabla_{1}^{2} C_{1}+\cdots+a_{7} \nabla_{1}^{7} C_{1}
$$


has the following coefficients in this case:

$$
\begin{gathered}
a_{0}=6 u u_{x}^{2}-6 u_{x x}^{2}-10 u_{x} v \\
-2 v_{x x x}+\left(6 u_{x} u_{x x}+2 v_{x x}\right) \frac{v_{x}}{v}, \\
a_{1}=4 u^{2} u_{x}-34 u_{x} u_{x x}-22 u v-14 v_{x x} \\
+\left(27 \lambda^{2}+4 u^{3}+6 u_{x}^{2}+16 u u_{x x}+12 v_{x}\right) \frac{v_{x}}{v}, \\
a_{2}=-27 \lambda^{2}-4 u^{3}-10 u_{x}^{2}-20 u u_{x x}-12 v_{x}+\frac{4 u u_{x} v_{x}}{v}, \\
a_{3}=13 u u_{x}-64 v+\left(9 u^{2}+35 u_{x x}\right) \frac{v_{x}}{v}, \\
a_{4}=-9 u^{2}-56 u_{x x}+\frac{21 u_{x} v_{x}}{v}, \\
a_{5}=-27 u_{x}+\frac{6 u v_{x}}{v}, \\
a_{6}=-6 u, \quad a_{7}=\frac{v_{x}}{v},
\end{gathered}
$$

where $v$ is given by (13).

For the matrix $X_{1}(12)$ with $\lambda=0$, we get quite a different situation. In this case, the dimension of the cyclic basis is five, not eight. The closure equation

$$
\begin{aligned}
\nabla_{1}^{5} C_{1}= & a_{0} C_{1}+a_{1} \nabla_{1} C_{1}+a_{2} \nabla_{1}^{2} C_{1} \\
& +a_{3} \nabla_{1}^{3} C_{1}+a_{4} \nabla_{1}^{4} C_{1}
\end{aligned}
$$

has the coefficients

$$
\begin{gathered}
a_{0}=-2 u u_{x}-2 u_{x x x}+\frac{2 u_{x} u_{x x}}{u}, \\
a_{1}=-4 u^{2}-8 u_{x x}+\frac{6 u_{x}^{2}}{u}, \\
a_{2}=-6 u_{x}, \quad a_{3}=-5 u, \quad a_{4}=\frac{u_{x}}{u} .
\end{gathered}
$$

For the matrix $X_{2}(14)$, which contains $u_{x}$, the characteristic matrix $C_{2}$ is computed in the following, more general, way:

$$
C_{2}=\frac{\partial X_{2}}{\partial u}-\nabla_{2}\left(\frac{\partial X_{2}}{\partial u_{x}}\right)=\left(\begin{array}{ccc}
0 & 0 & 0 \\
-1 & 0 & 0 \\
0 & 0 & 0
\end{array}\right)
$$

where the covariant derivative $\nabla_{2}$ is defined by the relation $\nabla_{2} A=D_{x} A-\left[X_{2}, A\right]$ with any $3 \times 3$ matrix $A$. The cyclic basis $C_{2}, \nabla_{2} C_{2}, \nabla_{2}^{2} C_{2}, \ldots, \nabla_{2}^{n-1} C_{2}$ for the matrix $X_{2}$ has the dimension $n=8$ if $\mu \neq 0$ and $n=5$ if $\mu=0$-the same dimensions as for the matrix $X_{1}$. The coefficients of closure equations in the case of $X_{2}$ are given by the expressions (19), after the replacement $\lambda^{2} \mapsto \mu^{2}$, for $\mu \neq 0$, and by the expressions (21) for $\mu=0$-the same expressions as for the matrix $X_{1}$. Taking into account that the dimensions of cyclic bases and the coefficients of closure equations are gauge invariants, we see that the only obstacle for the existence of a gauge transformation (16) we have found so far is the condition $\mu^{2}=\lambda^{2}$. This makes sense to try to find the matrix $G$ of (16) explicitly.

It is very convenient to make use of the fact that, under the gauge transformation (16), the characteristic matrix and its covariant derivatives transform as tensors [6,7]; namely,

$$
\nabla_{2}^{k} C_{2}=G\left(\nabla_{1}^{k} C_{1}\right) G^{-1}, \quad k=0,1,2, \ldots
$$

Denoting the elements of the matrix $G$ as $g_{i j}, i, j=1,2,3$, we find from the relation

$$
C_{2} G=G C_{1}
$$

that

$$
g_{11}=g_{13}=g_{33}=0, \quad g_{23}=g_{12} .
$$

Next, we find from the relation

$$
\left(\nabla_{2} C_{2}\right) G=G \nabla_{1} C_{1}
$$

that

$$
g_{21}=g_{22}=0, \quad g_{32}=-u g_{12} .
$$

Then, the relation

$$
\left(\nabla_{2}^{2} C_{2}\right) G=G \nabla_{1}^{2} C_{1}
$$

leads us to

$$
g_{31}=\lambda g_{12}, \quad \mu=\lambda \text {. }
$$

At this point, we can immediately conclude that the conditions $\lambda \neq 0$ and $g_{12} \neq 0$ hold necessarily because $\operatorname{det} G=$ $\lambda g_{12}^{3} \neq 0$. Finally, we get

$$
D_{x} g_{12}=D_{t} g_{12}=0
$$

directly from (16), that is, $g_{12}=c$ with any nonzero constant $c$, and obtain

$$
G=c\left(\begin{array}{ccc}
0 & 1 & 0 \\
0 & 0 & 1 \\
\lambda & -u & 0
\end{array}\right)
$$

With the natural choice of $c=1$ in (31), we have $\operatorname{det} G=\lambda$, and the inverse matrix $G^{-1}$ does not exist for $\lambda=0$. Of course, one can take $c=\lambda^{-1 / 3}$ and get $\operatorname{det} G=1$, but in this case the matrix $G$ does not exist for $\lambda=0$. As we have already pointed out above, the condition $\lambda \neq 0$ is necessary for the existence of the gauge transformation sought.

Consequently, the two considered ZCRs with the matrices $X$ and $T$ given by (12) and (14) are related to each other by the gauge transformation (16) if and only if $\mu=\lambda \neq 0$, and the corresponding matrix $G$ is given by (31), where one can take $c=1$ without loss of generality. One can see easily from (9), (11), and (31) that this gauge transformation corresponds to the transformation (6) between the Lax pairs considered in their operator form. Another way to see this consists in taking 
into account that $G$ in (31) with $c=1$ is identical to $X_{1}$ in (12), and therefore we have $\Phi \mapsto G \Phi=D_{x} \Phi$ in (9) owing to (7).

Let us note that it is a new, interesting, and quite surprising phenomenon that two ZCRs containing an essential parameter are related to each other by a gauge transformation for all values of the parameter except one value and no gauge transformation exists between those ZCRs for that single value of the parameter.

\section{Arbitrary Spectral Parameter}

Besides gauge transformations (9), there is a different-quite evident but rarely mentioned in the literature-nongauge type of equivalence transformations of ZCRs (8), namely, the transformation (10). Let us try to make use of a combination of transformations (9) and (10) to relate the two ZCRs given by (12) and (14) to each other.

The problem is to find a matrix $G$ such that

$$
\begin{aligned}
& X_{2}=G X_{3} G^{-1}+\left(D_{x} G\right) G^{-1}, \\
& T_{2}=G T_{3} G^{-1}+\left(D_{t} G\right) G^{-1},
\end{aligned}
$$

where

$$
X_{3}=-\widetilde{X}_{1}, \quad T_{3}=-\widetilde{T}_{1} .
$$

Since the gauge invariants of the cyclic basis in the case of $X_{3}$ coincide with the ones of $X_{1}$, we omit their consideration and proceed directly to the analysis of the relations $\nabla_{2}^{k} C_{2}=$ $G\left(\nabla_{3}^{k} C_{3}\right) G^{-1}, k=0,1,2, \ldots$, where $\nabla_{3}$ is defined by $\nabla_{3} A=$ $D_{x} A-\left[X_{3}, A\right]$ for any $3 \times 3$ matrix $A$, and $C_{3}=\partial X_{3} / \partial u=$ $-\widetilde{C}_{1}$. From the relation $C_{2} G=G C_{3}$, we find for the elements $g_{i j}$ of the matrix $G$ the following: $g_{11}=g_{12}=g_{32}=0$ and $g_{22}=-g_{13}$. Next, we find from the relation $\left(\nabla_{2} C_{2}\right) G=G \nabla_{3} C_{3}$ that $g_{21}=g_{23}=0$ and $g_{33}=-u g_{13}$. Then, the relation $\left(\nabla_{2}^{2} C_{2}\right) G=G \nabla_{3}^{2} C_{3}$ leads us to $g_{31}=g_{13}$ and $\mu=-\lambda$, where $g_{13} \neq 0$ in order to have $\operatorname{det} G \neq 0$. Finally, we get $D_{x} g_{13}=D_{t} g_{13}=0$ directly from (32), set $g_{13}=1$ without loss of generality, and obtain

$$
G=\left(\begin{array}{ccc}
0 & 0 & 1 \\
0 & -1 & 0 \\
1 & 0 & -u
\end{array}\right)
$$

Consequently, the two considered ZCRs with the matrices $X$ and $T$ given by (12) and (14) are related to each other by the combination of transformations (32) and (33) if and only if $\mu=-\lambda$, and the corresponding matrix $G$ is given by (34). The case of zero spectral parameter is included now. Let us note that we were forced to use the nongauge transformation (10), which is evidently a counterpart of the transformation (5), in order to cover the case of zero spectral parameter, because the two studied ZCRs belong to two distinct classes of gauge equivalence if the spectral parameter is zero.

\section{Conclusion}

In this paper, using the method of gauge-invariant description of zero-curvature representations (ZCRs) and the method of cyclic bases of ZCRs, we have shown that the new Lax pair of the Sawada-Kotera equation, discovered recently by Hickman, Hereman, Larue, and Göktas, and the wellknown old Lax pair of this equation, considered in the form of ZCRs, are gauge equivalent to each other if and only if the spectral parameter is nonzero, while for zero spectral parameter a nongauge transformation is required. As a byproduct, we have obtained an interesting example of two ZCRs which share the same set of gauge invariants but cannot be related to each other by a gauge transformation.

\section{Conflict of Interests}

The author declares that there is no conflict of interests regarding the publication of this paper.

\section{Acknowledgments}

The author is grateful to Ziemowit Popowicz, Takayuki Tsuchida, Allan Fordy, and anonymous reviewers for valuable comments.

\section{References}

[1] M. Hickman, W. Hereman, J. Larue, and Ü. Göktaş, "Scaling invariant Lax pairs of nonlinear evolution equations," Applicable Analysis, vol. 91, no. 2, pp. 381-402, 2012.

[2] K. Sawada and T. Kotera, "A method for finding $N$-soliton solutions of the K.D.V. equation and K.D.V.-like equation," Progress of Theoretical Physics, vol. 51, no. 5, pp. 1355-1367, 1974.

[3] P. J. Caudrey, R. K. Dodd, and J. D. Gibbon, "A new hierarchy of Korteweg-de Vries equations," Proceedings of the Royal Society $A$, vol. 351, no. 1666, pp. 407-422, 1976.

[4] R. K. Dodd and J. D. Gibbon, "The prolongation structure of a higher order Korteweg-de Vries equation," Proceedings of the Royal Society of London A, vol. 358, no. 1694, pp. 287-296, 1978.

[5] A. P. Fordy and J. Gibbons, "Factorization of operators. I. Miura transformations," Journal of Mathematical Physics, vol. 21, no. 10, pp. 2508-2510, 1980.

[6] M. Marvan, "On zero-curvature representations of partial differential equations," in Differential Geometry and its Applications, O. Kowalski and D. Krupka, Eds., vol. 1, pp. 103-122, Silesian University in Opava, Opava, Czech Republic, 1993.

[7] S. Y. Sakovich, "On zero-curvature representations of evolution equations," Journal of Physics A: Mathematical and General, vol. 28, no. 10, pp. 2861-2869, 1995.

[8] S. Y. Sakovich, "Cyclic bases of zero-curvature representations: five illustrations to one concept," Acta Applicandae Mathematicae, vol. 83, no. 1-2, pp. 69-83, 2004. 


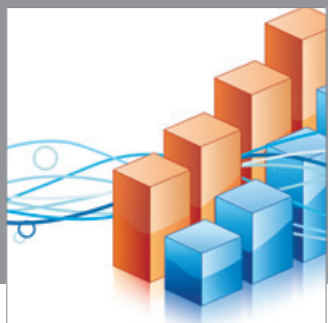

Advances in

Operations Research

mansans

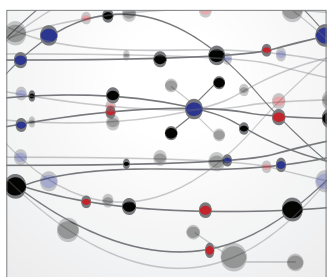

The Scientific World Journal
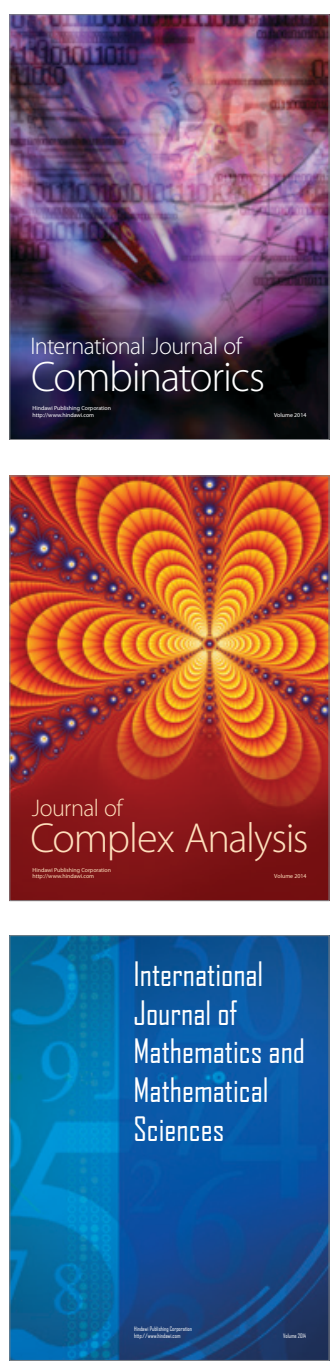
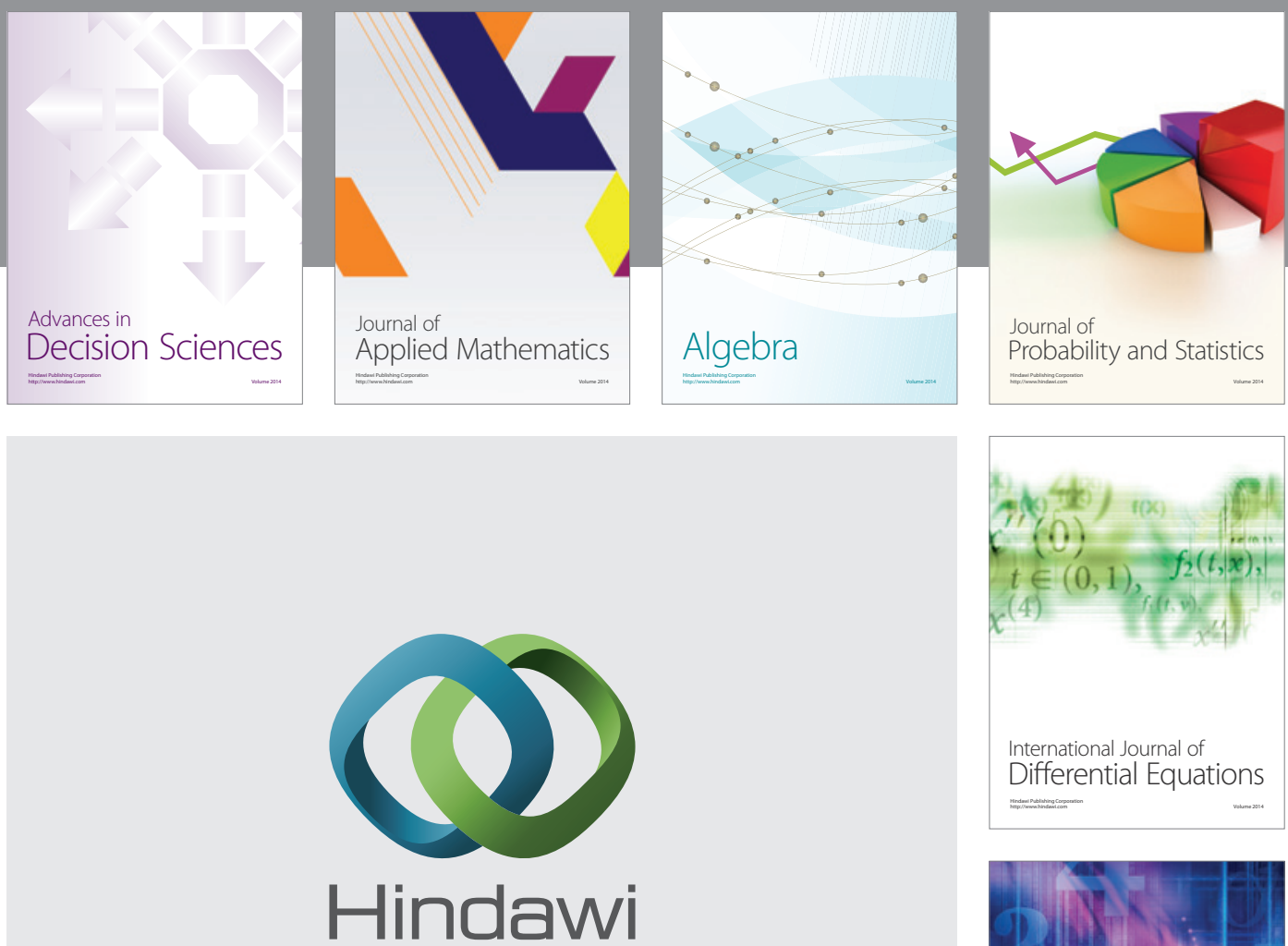

Submit your manuscripts at http://www.hindawi.com
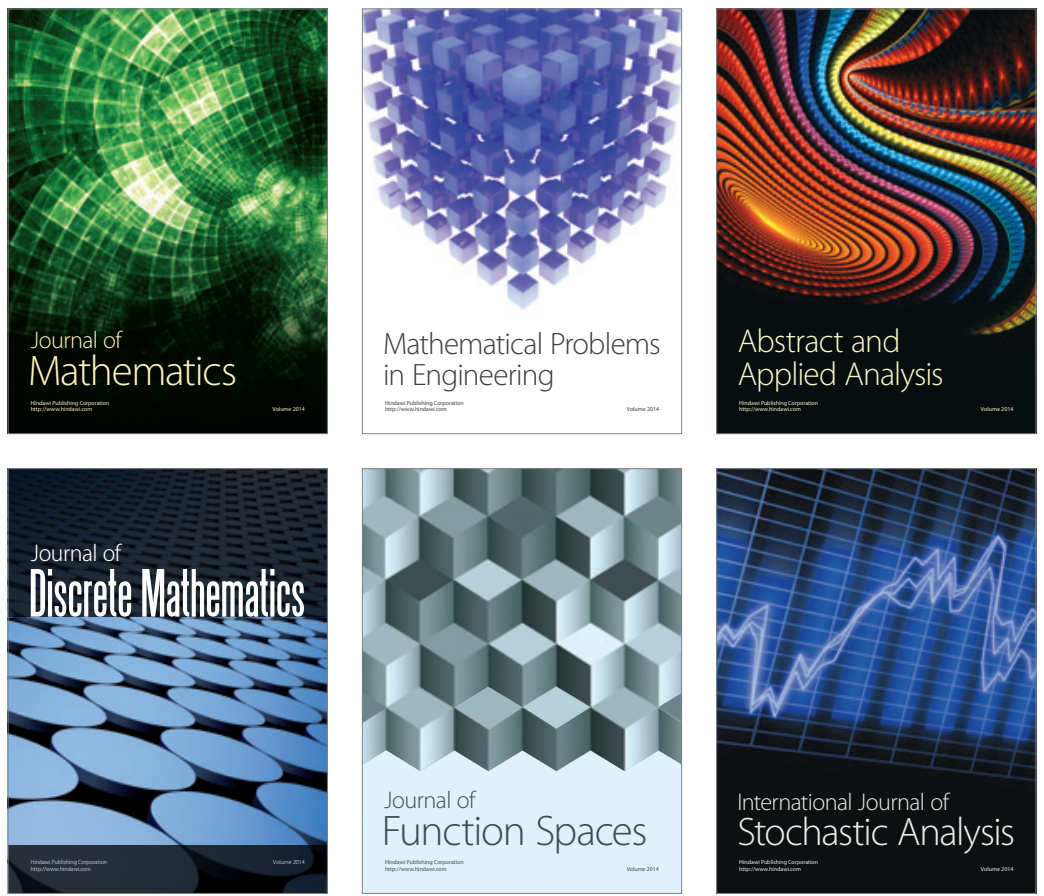

Journal of

Function Spaces

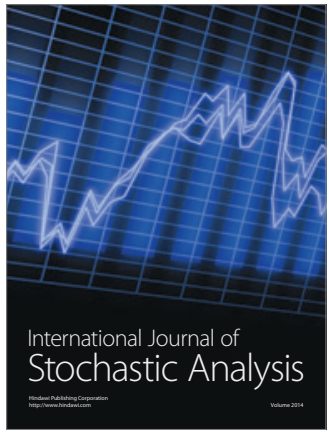

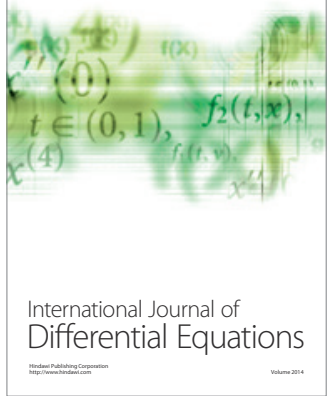
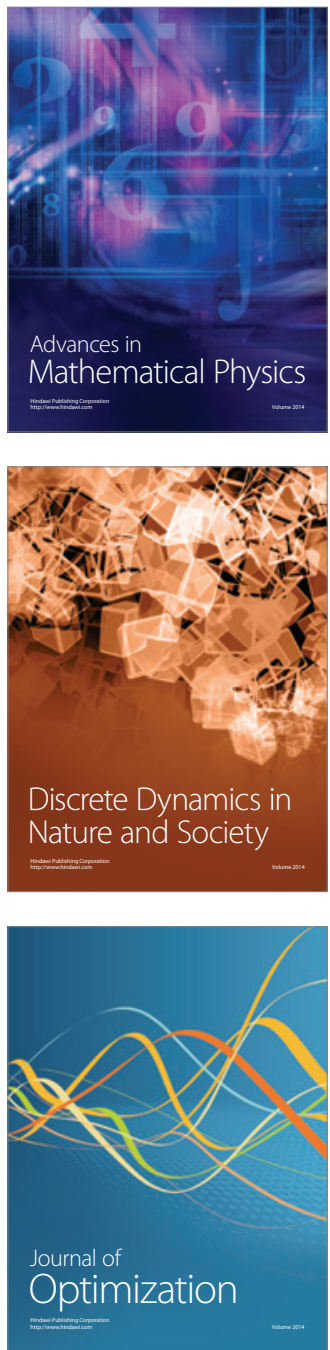